\title{
Enxaqueca e Estresse em Mulheres no Contexto da Atenção Primária ${ }^{1}$
}

\author{
Luciana Leonetti Correia \\ Universidade Federal da Grande Dourados \\ Maria Beatriz Martins Linhares ${ }^{2}$ \\ Faculdade de Medicina de Ribeirão Preto
}

\begin{abstract}
RESUMO - O presente estudo teve por objetivo verificar a associação entre enxaqueca e estresse em mulheres, assim como examinar o melhor modelo de predição da enxaqueca, considerando variáveis pessoais e do contexto ambiental. A enxaqueca foi identificada pelo Teste de Cefaleia em 75 mulheres sem antecedentes psiquiátricos. $\mathrm{O}$ estresse foi avaliado por meio do Inventário de Sintomas de Stress para adultos. Paralelamente, foram avaliados: eventos vitais, nível socioeconômico e características da amostra. Verificou-se que 55\% das mulheres apresentaram enxaqueca e 59\% sintomas de estresse. O modelo de predição identificou que o estresse foi o único preditor da enxaqueca em mulheres. Os achados mostram associação entre enxaqueca e estresse, a qual precisa ser levada em conta na assistência à saúde da mulher.
\end{abstract}

Palavras-chave: enxaqueca, estresse, mulheres

\section{Migraine and Stress in Women in the Primary Health Care}

\begin{abstract}
The aim of the present study was to verify the relation between migraine and stress, and to identify the best predictor model for migraine, considering personal and environment variables. Migraine was identified applying the Test of the Brazilian Headache Society to 75 women without psychiatric history. Stress was identified by the Stress Symptom for Adults Inventory. Simultaneously, life events, socioeconomic level, and sample characteristics were assessed. According to the results, $55 \%$ of women presented migraine and $59 \%$ showed stress. The prediction model revealed that the presence of stress was the only predictor of the presence of migraine in women. These findings suggest an association between migraine and stress, which should be considered in the health assistance of women.
\end{abstract}

Keywords: migraine, stress, women

Segundo a Organização Mundial de Saúde (OMS, 2006), a cefaleia apresenta-se como a segunda queixa mais comum de dor. Estima-se que mais da metade da população mundial apresente algum tipo de cefaleia em determinada fase da vida (Nobre, 2006).

De acordo com a classificação da International Headache Society (2008), as cefaleias e as dores craniofaciais são classificadas como primárias ou secundárias. As cefaleias primárias caracterizam-se pela ausência de anormalidades anatomopatológicas e não são decorrentes de doenças orgânicas específicas de origem intracraniana ou sistêmica (Göbel, 2001). As cefaleias incluem a enxaqueca ou migrânea com e sem aura, a cefaleia do tipo tensão ou tensional, a cefaleia em salvas, cefaleias trigêmino-autonômicas (incluindo as hemicranias paroxísticas) e cefaleias diversas não associadas à lesão estrutural (cefaleia idiopática em facadas ou pontadas, cefaleia da tosse, cefaleia do esforço físico, cefaleia associada à atividade sexual, cefaleia hípnica, cefaleia em trovoada ou "thunderclap headache", hemicrania contínua e cefaleia persistente e diária desde o início). As

1 Apoio: CAPES

2 Endereço para correspondência: Lab. de Pesquisa Prevenção de Problemas de Desenvolvimento e Comportamento da Criança, Av. Tenente Catão Roxo 2650/ sala 52, Prédio da Saúde Mental- Faculdade de Medicina de Ribeirão Preto, Universidade de São Paulo, Campus Universitário Monte Alegre, Ribeirão Preto, SP, Brasil. CEP: 14048900.E-mail: linhares@fmrp.usp.br cefaleias secundárias, por sua vez, são decorrentes de lesões identificadas no segmento cefálico ou de afecções sistêmicas, passíveis de serem investigadas com métodos clínicos, pois ocorrem como sintomas de outras doenças orgânicas mais graves e podem ser atribuídas a: traumatismo craniano e ou cervical, doenças vasculares craniana ou cervical, doença intracraniana não vascular, uso de substâncias químicas, drogas, medicamentos ou à sua supressão, infecção, transtornos da homeostase, transtornos de estruturas cranianas e cervicais e transtornos psiquiátricos (Göbel, 2001).

As cefaleias podem também ser relacionadas, segundo a International Headache Society (2008), a dor facial neuropática, dor facial central e cefaleias não classificáveis ou não especificáveis. As cefaleias mais frequentes na população, em geral, são as enxaquecas ou migrâneas e as cefaleias do tipo tensional (Puccini \& Bresolin, 2003).

Em um estudo de revisão sobre estudos epidemiológicos mundiais em amostras não referidas clinicamente, a prevalência mundial de cefaleia na população adulta foi de $46 \%$, sendo que a cefaleia do tipo tensional foi a mais prevalente $(42 \%)$, seguida pela enxaqueca $(11 \%)$ (Stovner et al., 2007). Entretanto, deve-se identificar os fatores que podem influenciar esses índices de prevalência de cefaleia na população, dependendo da definição e do diagnóstico de cefaleia utilizado, da metodologia e da coleta de dados, os estudos podem apresentar diferentes prevalências de 
cefaleia (Kienbacher et al., 2006). Além disso, estudos têm identificado que a prevalência de cefaleia em relação ao sexo pode variar conforme a faixa etária (Aromaa, Rautava, Helenius, \& Sillanpää, 1998; Campo, Comer, Jansen-Mcwilliams, Gardner, \& Kelleher, 2002; Fearon \& Hotopf, 2001; Speciali, 2003; Bugdayci et al., 2005). Até os 7 anos de idade, a frequência de cefaleia é a mesma para ambos os sexos, entretanto, na adolescência há um aumento da frequência de cefaleia, sendo que as meninas passam a apresentá-la com maior frequência do que os meninos. Por fim, alguns fatores relacionados a particularidades de cada país, tais como condições sócio-demográficas, climáticas, aspectos culturais e genéticos podem afetar a prevalência de cefaleia (Stovner et al., 2007).

Estudos acerca da epidemiologia da cefaleia no Brasil encontraram altas prevalências na população brasileira, com variação entre $43 \%$ a $93 \%$, quando comparadas a estimativas de prevalências no mundo, a qual é da ordem de $46 \%$ na população adulta (Domingues, Aquino, Santos, Silva, \& Kuster, 2006; Fernandes, 2004; Queiroz, Barea, \& Blank, 2006; Queiroz et al., 2009; Stovner et al., 2007).

Por meio de levantamento epidemiológico, Fernandes (2004) verificou a prevalência de cefaleia em pessoas residentes na cidade de Ribeirão Preto, por meio de entrevistas telefônicas. Dos 1.230 indivíduos, 552 (44,9\%) queixavam-se de cefaleia no momento do estudo. Dessa amostra, $383(69,4 \%)$ eram mulheres, sendo que 29,7\% destas relataram presença de cefaleia. Além disso, foi verificada associação positivamente significativa entre ser mulher e ter cefaleia, dado este que é sustentado por outros estudos devido a fatores clínicos relacionados a hormônios femininos (Bugdayci et al, 2005; Rasmussen, 2001). Entretanto, deve-se ter cautela em relação à generalização desse dado, especialmente em populações nas quais há uma preponderância do sexo feminino em idade adulta (Stewart, Lipton, Celentano, \& Reed, 1992).

Considerando os tipos de cefaleia primária, a enxaqueca ou migrânea caracteriza-se por manifestação álgica de moderada/ forte intensidade, frequentemente interferindo nas atividades cotidianas, acompanhada por sintomas gastrointestinais (náuseas e vômitos), fotofobia, fonofobia e, ocasionalmente, por manifestações neurológicas transitórias - como hemianopsia, paresia e ataxia - que caracterizam a aura (Puccini \& Bresolin, 2003). O diagnóstico de enxaqueca para adultos prevê a ocorrência de mais de cinco crises, com duração entre quatro (duas horas para menores de 15 anos) e 72 horas, além de critérios relativos à localização, intensidade da dor e presença de náuseas ou vômitos, fotofobia e fonofobia (Headache Classification Subcommittee of the International Headache Society, 2004). A enxaqueca ainda pode ser classificada em enxaqueca com ou sem aura e outros tipos de enxaqueca.

De acordo com o estudo de Queiroz et al. (2009), realizado em 27 estados brasileiros, por meio de entrevistas pelo telefone, com 3.848 pessoas na faixa etária entre 18 a 79 anos, verificou-se que a prevalência estimada do diagnóstico de enxaqueca foi de $16 \%$ da amostra total. A prevalência de enxaqueca foi maior na região Sudeste $(20,5 \%)$, seguida pelas regiões Sul $(16,4 \%)$, Nordeste $(13,6 \%)$, Centro-Oeste $(9,5 \%)$ e Norte $(8,5 \%)$. A migrânea foi mais prevalente em mulheres, de baixa renda e que não praticavam exercícios físicos regularmente. O estudo de Queiroz et al. (2006) encontrou que a prevalência de enxaqueca foi de $40,3 \%$ em uma amostra randomizada da população de Florianópolis, sendo mais prevalente em mulheres, na faixa etária dos 35 aos 44 anos. Domingues et al. (2006) verificou que, em uma população rural do Espírito Santo, atendida pelo Programa de Saúde da Família, a prevalência da enxaqueca foi de 55,5\% dos indivíduos.

Há inúmeros fatores que se relacionam a possíveis causas da enxaqueca. Dentre estes, destaca-se o estresse, uma vez que, desde pequenos acontecimentos do dia a dia a eventos com alto grau de estresse podem contribuir para o início da enxaqueca (Galego, 2006). Além disso, estudos mostram que o estresse pode aumentar a sensibilidade à dor e afetar todo o processamento da dor no Sistema Nervoso Central (Cathcart, Winefield, Lushington, \& Rolan, 2010).

Atualmente, o estresse é visto pela Organização Mundial de Saúde como um dos principais distúrbios contemporâneos, atingindo grande contingente de pessoas e associado a uma enorme gama de doenças, ao absenteísmo e a elevados custos na assistência médica (Lipp, 2004). Os estudos iniciais sobre o estresse procuravam mensurá-lo por meio da avaliação de eventos de vida estressantes. Holmes e Rahe (1967) sugeriram que o nível de estresse pode ser medido indiretamente por meio da avaliação de grandes fatores estressantes que tenham ocorrido na vida da pessoa nos últimos meses. De acordo com Kanner, Coyne, Schaefer e Lazarus (1981), os pequenos aborrecimentos diários também devem ser verificados, juntamente com os grandes estressores, uma vez que, os primeiros podem possuir um efeito cumulativo no organismo. Posteriormente, Lazarus e Folkman (1984) demonstraram que não são os eventos de vida em si que levam ao estresse patológico, mas a avaliação que o indivíduo faz das situações por que passa e que as diferenças ambientais, orgânicas e psicológicas têm um importante papel mediador.

$\mathrm{O}$ estresse emocional é um dos principais fatores desencadeantes da enxaqueca, como também está relacionado com a duração e piora das crises. Segundo Lipp e Malagris (2001, p. 477)

o estresse é definido como uma reação do organismo, com componentes físicos e/ou psicológicos, causada pelas alterações psicofisiológicas, que ocorrem quando uma pessoa se confronta com uma situação que, de um modo ou de outro, a irrite, amedronte, excite, confunda ou mesmo que a faça imensamente feliz.

Portanto, a resposta de estresse deve ser entendida como um processo que, em sua fase inicial, pode ser benéfico e positivo. De acordo com a Síndrome de Adaptação Geral, descrita por Selye (1952), essa resposta inicial corresponde à fase de alerta e, é quando o organismo produz adrenalina que fornece ânimo, vigor e energia para enfrentar os eventos adversos. Entretanto, o organismo não consegue permanecer alerta por muito tempo, esgotando a capacidade de adaptação do mesmo (Lipp, 2000).

Dessa forma, se os fatores estressantes persistirem, o organismo tentará restabelecer o equilíbrio interno e entrará em uma segunda fase, a de resistência. Caso os fatores estressantes continuem persistindo, em frequência 
ou intensidade, o organismo se aproximará da terceira fase, a de quase-exaustão. Nessa fase, inicia-se um processo de adoecimento e os órgãos que possuírem maior vulnerabilidade genética ou adquirida podem apresentar sinais de deterioração. Sendo assim, quando o estressor perdura ou quando há a presença simultânea de outros estressores, o processo de estresse evolui para a última fase que é a de exaustão e que corresponde ao aparecimento de doenças graves, tais como enfarte, úlcera, psoríase e depressão. Mulheres na faixa etária dos 18 aos 72 anos com enxaqueca apresentaram sintomas de estresse, quando avaliadas por meio do ISSL (Mascella, 2011).

De acordo com Lipp (2005), o estresse e a dor, seja ela qual for, estão entre os fatores que mais aborrecem, debilitam e invalidam o ser humano para o cumprimento do trabalho, para viver com qualidade e para o usufruto da felicidade. Em muitos casos, o estresse e a dor encontram-se intimamente associados, uma vez que, o estresse pode aparecer tanto como desencadeador ou cooperador para a etiologia da dor ou ocorrer em consequência da dor. No entanto, alguns estudos que analisaram a relação entre estresse e enxaqueca apresentaram resultados ainda inconclusivos (Bosco, 2006; Guidetti \& Galli, 1998; Karwautz, Wober-Bingol, \& Wober, 1993). Além disso, até onde se sabe, os estudos não controlaram os eventos vitais e antecedentes psiquiátricos que podem ter relação com o estresse.
O presente estudo teve por objetivo verificar a associação entre enxaqueca em mulheres e estresse, assim como examinar o melhor modelo de predição da enxaqueca, considerando variáveis pessoais e do contexto ambiental.

\section{Método}

\section{Participantes}

Inicialmente, foi realizado um levantamento dos registros das mulheres pertencentes a famílias cadastradas nos cinco Núcleos do Programa de Saúde da Família (PSF), vinculados a Faculdade de Medicina de Ribeirão Preto da Universidade de São Paulo (FMRP-USP). Esses Núcleos são Centros de Atenção Primária à Saúde e, portanto, a amostra deste estudo foi extraída da comunidade (população não-referida) e não do contexto de atendimento clínico especializado.

O levantamento inicial foi obtido por meio dos dados dos agentes de saúde dos Núcleos do PSF acerca das mulheres as quais eles eram responsáveis pelo atendimento no período de 28/03/2006 a 14/04/2006. Foram encontrados 269 cadastros de mulheres nos Núcleos do PSF. Destas, 162 mulheres não foram localizadas em suas residências. Foram localizadas e visitadas 107 mulheres, das quais 23

Tabela 1. Características da amostra $(\mathrm{N}=75)$

\begin{tabular}{|c|c|}
\hline Características das mulheres & Valores \\
\hline Idade (anos) - média (desvio padrão) & $28,89( \pm 5,86)$ \\
\hline $\begin{array}{l}\text { União civil - frequência (porcentagem) } \\
\text { União estável } \\
\text { Separada/ divorciada } \\
\text { Solteira } \\
\text { Viúva }\end{array}$ & $\begin{array}{l}60(80 \%) \\
8(11 \%) \\
5(7 \%) \\
2(2 \%)\end{array}$ \\
\hline Número de filhos - média (desvio padrão) & $2,49( \pm 1,46)$ \\
\hline Escolaridade (anos) - média (desvio padrão) & $7,63( \pm 3,65)$ \\
\hline $\begin{array}{l}\text { Escolaridade (grau)- frequência (porcentagem) } \\
\text { Fundamental } 1 \text { (incompleto) } \\
\text { Fundamental } 1 \text { (completo) e Fundamental } 2 \text { (incompleto) } \\
\text { Fundamental } 2 \text { (completo) e Médio Incompleto } \\
\text { Médio (completo) e Superior Incompleto } \\
\text { Superior Completo }\end{array}$ & $\begin{array}{l}10(13 \%) \\
26(35 \%) \\
20(27 \%) \\
15(20 \%) \\
4(5 \%)\end{array}$ \\
\hline $\begin{array}{l}\text { Ocupação profissional- frequência (porcentagem) } \\
\text { Do lar } \\
\text { Trabalhadora }\end{array}$ & $\begin{array}{l}43(56 \%) \\
32(44 \%)\end{array}$ \\
\hline $\begin{array}{l}\text { Nível da qualificação ocupacional(a) - frequência (porcentagem) } \\
\text { Não qualificada } \\
\text { Qualificação inferior } \\
\text { Qualificação superior }\end{array}$ & $\begin{array}{l}27(84 \%) \\
2(6 \%) \\
3(10 \%)\end{array}$ \\
\hline $\begin{array}{l}\text { Classificação socioeconômica }(b) \\
\text { Baixo } \\
\text { Médio }\end{array}$ & $\begin{array}{l}58(77 \%) \\
17(23 \%)\end{array}$ \\
\hline
\end{tabular}

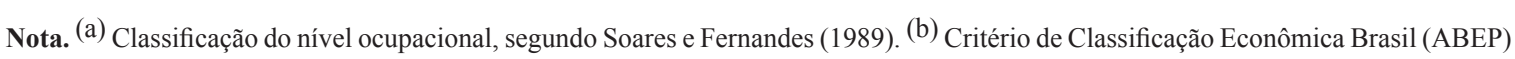


não aceitaram participar do presente estudo. Sendo assim, 84 mulheres foram encontradas e foram aplicados os critérios de exclusão (problemas psiquiátricos e deficiências). Essas exclusões foram relevantes, na medida em que o foco do estudo eram mulheres não-clínicas e não-referidas. As mulheres, portanto, não deveriam ser a priori pertencentes a amostras identificadas; as queixas de enxaqueca e o estresse não deveriam estar associados a transtornos psiquiátricos ou deficiências identificadas previamente.

Oito mulheres foram excluídas por apresentarem antecedentes psiquiátricos, os quais foram avaliados pela SCID Não-Paciente, Entrevista Clínica Estruturada para DSM III-R (Spitzer, Willians, Gibbon, \& First, 1989, com tradução de Del Ben, 1995). Uma mulher também foi excluída por apresentar déficit auditivo, o que a impediu de participar das avaliações. Portanto, a amostra final do presente estudo foi composta por 75 mulheres.

De acordo com a Tabela 1, as mulheres, na amostra total, na maioria eram jovens, com idade média de 28 anos $( \pm$ $5,86)$, viviam em união civil estável, possuíam, em média, dois filhos e haviam cursado o ensino fundamental completo. Em relação à ocupação profissional, a maior parte dessas mulheres não trabalhava e as que trabalhavam exerciam atividades não qualificadas. De acordo com o Critério de Classificação Econômica Brasil (ABEP), a maior parte das mulheres pertencia a famílias correspondentes a um perfil de classe social menos favorecida economicamente.

\section{Instrumentos}

A SCID Não-Paciente, Entrevista Clínica Estruturada para DSM III R (Spitzer et al., 1989) consiste em um roteiro semiestruturado de entrevista, norteador de uma investigação clínica de distúrbios psiquiátricos, designada para uso com indivíduos não identificados como pacientes psiquiátricos. Esse instrumento deve ser administrado por um profissional de Saúde Mental treinado e familiarizado com a classificação e os critérios diagnósticos do DSM-III-R (Del Ben, 1995). O instrumento fornece um julgamento clínico sobre o histórico de transtornos psiquiátricos e foi utilizado, sem finalidade diagnóstica, para excluir mulheres que apresentavam histórico psiquiátrico.

Foi utilizado o teste elaborado pela Sociedade Brasileira de Cefaleia (Speciali, 2003), o qual verifica a presença de sintomas relacionados à enxaqueca. $\mathrm{O}$ teste é composto por quatro blocos que correspondem à intensidade (avaliada por quatro itens), características da dor (avaliada por dois itens), duração e frequência da enxaqueca, ambas avaliadas por um item cada. A intensidade, as características e a duração da enxaqueca apresentam duas alternativas de resposta ( $\operatorname{sim}$ ou não). A frequência da enxaqueca foi avaliada pelo número de crises mensais, em cinco alternativas de respostas $(0 ; 1 ; 2$ a 4 ; 5 a $8 ;>9)$. O critério para identificar a presença de sintomas de enxaqueca considerou, no último mês, a presença de pelo menos duas ou mais respostas sim no item de intensidade, uma ou mais resposta sim no item de duração e uma resposta sim no item de característica, além de uma a quatro crises de enxaqueca mensais no item de frequência. O teste é validado para detecção da enxaqueca e reconhecido pela Sociedade Brasileira de Cefaleia.

O Inventário de Sintomas de Stress para adultos de Lipp - ISSL (Lipp, 2000) é um instrumento que permite realizar um diagnóstico preciso de estresse, determinar a fase na qual a pessoa se encontra (alerta, resistência, quase-exaustão ou exaustão) e área predominante de manifestação desse estresse (física ou psicológica). É um instrumento de fácil aplicação e de curto tempo de administração (cerca de 10 minutos). O ISSL inclui 37 itens de natureza somática e 19 de natureza psicológica, sendo que os sintomas diferem em sua intensidade e gravidade, considerando três períodos: as últimas 24 horas, a última semana e o último mês.

A presença de cada sintoma equivale a um ponto. A soma dessa pontuação fornecerá um escore bruto e uma porcentagem específica dos sintomas físicos e psicológicos e, de acordo com os critérios especificados por Lipp (2000), corresponderá à fase de estresse na qual o indivíduo se encontra. Para cada fase, há uma variação do escore bruto e das porcentagens. Na fase de alerta, o escore bruto varia de sete a 15 e com porcentagens de 11 a 100\%; na fase de resistência, encontra-se um escore bruto variando de quatro a nove e porcentagens de 8 a 50\%; a fase de quase exaustão apresenta uma variação de 10 a 15 no escore bruto e de 58 a $100 \%$ e, na fase de exaustão, o escore bruto varia de nove a 23 e as porcentagens de 7 a $100 \%$. Dentro de cada fase, quanto maior o escore bruto e as porcentagens, maior a gravidade do estresse. A confiabilidade desse instrumento, por meio do alfa de Cronbach, foi de 0,91 (Lipp, 2000).

A Escala de Eventos Vitais (Savoia, 1999), por sua vez, consiste na verificação da presença ou não de 26 itens relacionados a mudanças inesperadas no ambiente social ocorridos nos últimos 12 meses, sem a atribuição de algum valor de julgamento clínico. Trata-se de uma medida descritiva. De acordo com Savoia (1999), esses itens são agrupados em categorias, a saber: trabalho (mudança de trabalho, dificuldades com a chefia, reconhecimento profissional e perda de emprego), perda de suporte social (morte de um amigo, morte de alguém da família e morte do cônjuge), família (doença na família, separação, casamento e gravidez), mudanças no ambiente (mudança de casa, mudança de escola e mudança do número de pessoas morando em casa), dificuldades pessoais (problemas de saúde, mudança de hábitos pessoais, mudanças de atividades sociais, mudanças de atividades religiosas, mudanças de atividades recreativas, acidentes, dificuldades sexuais e aposentadoria) e finanças (dívidas e perdas financeiras). Os resultados são obtidos a partir da soma dos itens e fornecem uma pontuação do número de eventos vitais. Quanto mais alta a soma, mais eventos vitais adversos.

Por fim, o Questionário da Associação Brasileira de Estudos Populacionais- ABEP (http://www.abep.org/) trata-se de um questionário para caracterização econômica da amostra. Este é formado por questões acerca do grau de instrução do chefe da família e da presença de eletrodomésticos, eletroeletrônicos, automóvel e empregada mensal na residência da família. A soma do número de itens fornece uma pontuação, que categoriza a família da mulher em relação a sua classe econômica, conforme o "Critério de Classificação Econômica Brasil” (CCEB) da ABEP (2013). 
De acordo com a classe econômica, estima-se a renda média bruta familiar, como segue a descrição: Classe A - varia de 35 a 46 pontos na escala, sendo que a renda média bruta familiar mensal estimada é de R \$ 9.263,00; Classe B1- varia de 29 a 34 pontos, renda média bruta familiar mensal estimada de R \$ 5.241,00; Classe B2 - varia de 23 a 28 pontos, renda média bruta familiar mensal estimada de $\mathrm{R} \$ 2.654,00$; Classe C1 - varia de 18 a 22 pontos, renda média bruta familiar mensal estimada é de R \$1.685,00; Classe C2 - varia de 14 a 17 pontos, renda média bruta familiar estimada é de R\$ $1.147,00$ e as Classes D/E - variam de 0 a 13 pontos, renda média bruta familiar mensal estimada é de $\mathrm{R} \$ 776,00$.

\section{Procedimentos de coleta de dados}

O presente projeto foi aprovado pelo Comitê de Ética em Pesquisa do Centro de Saúde Escola (CSE) da Faculdade de Medicina de Ribeirão Preto (FMRP - USP). As mulheres foram convidadas a participar do presente estudo por meio de contato telefônico, contato pessoal nas consultas médicas realizadas nos Núcleos ou em visitas domiciliares. Após a aceitação das mulheres para participar do estudo e da assinatura do Termo de Consentimento Livre e Esclarecido, procedeu-se à coleta de dados por meio de visitas domiciliares agendadas. A coleta de dados incluiu a aplicação da SCID Não-paciente para identificar os antecedentes psiquiátricos nas mulheres. Caso fosse verificada a ausência desses antecedentes, prosseguia-se a coleta e, na sequência, eram aplicados os seguintes instrumentos: o Questionário ABEP, o Teste de Cefaleia, a Escala de Eventos Vitais e o Inventário de Sintomas de Stress para adultos de Lipp (2000).

\section{Análise de dados}

A preparação para a análise dos dados foi realizada de acordo com as normas dos respectivos instrumentos. A classificação socioeconômica fornecida pelo Questionário da Associação Brasileira de Estudos Populacionais- ABEP foi categorizada de acordo com a renda familiar mensal estimada em três níveis: a) baixo: que abrangeu as classes E, D e C2, compreendendo uma renda familiar mensal estimada entre $\mathrm{R} \$$ 776,00 a R $\$ 1.147,00$; b) médio: que abrangeu as classes $\mathrm{C} 1$ e B2, compreendendo uma renda familiar mensal estimada entre $R \$ 1.685,00$ a $R \$ 2.654,00$ e c) alto: abrangendo as classes B1 e A, com uma renda familiar mensal estimada entre $\mathrm{R} \$ 5.241,00$ a $\mathrm{R} \$ 9.263,00$.

Os dados foram submetidos à análise de estatística descritiva em termos de média e desvio padrão para as variáveis contínuas, e frequência e porcentagem para as variáveis categóricas. A fim de identificar o melhor modelo de predição de enxaqueca em mulheres, utilizou-se da análise de regressão logística. Primeiramente, foi realizada análise de correlação de Spearman entre as seguintes variáveis: a) pessoais (idade, estado civil, nível de escolaridade e socioeconômico); b) do contexto ambiental (número de eventos vitais); c) presença ou não de sintomas de estresse nas mulheres. As variáveis que apresentaram associações estatisticamente significativas foram incluídas no modelo e consideradas como variáveis preditoras para a variável predita de presença ou não de enxaqueca em mulheres (presença $=1$; ausência=zero). No tratamento estatístico dos dados foi utilizado o Statistical Package for Social Sciences (SPSS ${ }^{\circ}$, versão 17.0; Chicago, IL, USA). O nível de significância adotado no presente estudo foi de $5 \%$.

\section{Resultados}

A Tabela 2 apresenta todas as categorias da Escala de Eventos Vitais e apenas os eventos vitais de maior frequência (acima de 30\%) correspondentes a essas categorias, nas mulheres estudadas.

Tabela 2. Número e tipos de eventos vitais na história de vida das mulheres

\begin{tabular}{lc}
\hline \multicolumn{1}{c}{ Eventos vitais } & Valores \\
\hline Número de Eventos - Média (Desvio Padrão) & $6( \pm 3,68)$ \\
$\begin{array}{l}\text { Tipos de eventos vitais - frequência (\%) } \\
\text { Finanças } \\
\quad \text { Dívidas } \\
\begin{array}{l}\text { Família } \\
\text { Gravidez }\end{array} \\
\begin{array}{l}\text { Perda de suporte social } \\
\quad \text { Morte de alguém da família }\end{array} \\
\begin{array}{l}\text { Trabalho } \\
\quad \text { Perda de emprego }\end{array} \\
\begin{array}{l}\text { Mudanças de ambiente } \\
\text { Mudanças do número de pessoas morando em }\end{array} \\
\begin{array}{l}\text { casa } \\
\hline\end{array}\end{array}$ 26(35\%) \\
\hline
\end{tabular}

A média do número de eventos vitais ocorridos nos últimos 12 meses na amostra total foi em torno de seis eventos. O evento vital mais frequente, ocorrido nos últimos 12 meses, foi o de "dívidas", dentro da categoria finanças. Na categoria família, o evento "gravidez" foi o mais frequente. O evento "morte de alguém da família" foi o mais frequente na categoria perda de suporte social. O evento "perda de emprego" foi o mais frequente na categoria trabalho e na categoria mudanças no ambiente, o evento mais frequente foi o de "mudança do número de pessoas morando em casa".

A Tabela 3 apresenta os dados relativos à presença de enxaqueca e estresse nas mulheres.

Tabela 3. Enxaqueca e estresse nas mulheres

\begin{tabular}{lc}
\hline \multicolumn{1}{c}{ Eventos Vitais } & Frequência (\%) \\
\hline Presença de enxaqueca & $41(55 \%)$ \\
Presença de estresse & $44(59 \%)$ \\
Fase do estresse & \\
$\quad$ Alerta & $2(4 \%)$ \\
Resistência & $38(86 \%)$ \\
Quase-exaustão & $3(7 \%)$ \\
Sintomatologia do estresse & $1(3 \%)$ \\
Psicológico & $26(59 \%)$ \\
Físico & $18(41 \%)$ \\
\hline
\end{tabular}


Na Tabela 3, em relação à presença de enxaqueca, verificou-se que na amostra total, 41 (55\%) mulheres relataram sintomas de enxaqueca. Em relação ao estresse, mais da metade das mulheres (59\%) relatou sintomas relacionados à presença de estresse. De acordo com o ISSL, a maioria das mulheres encontrava-se na fase de resistência do estresse, que corresponde a uma fase na qual há um agravamento dos sintomas de estresse. Além disso, a maior parte desses sintomas eram predominantemente psicológicos do que físicos.

Em relação ao modelo de predição da presença de enxaqueca em mulheres, verificou-se associações entre a presença de sintomas de enxaqueca e a presença de estresse $(\mathrm{r}=0,43 ; \mathrm{p}=0,0001)$ e o maior número de eventos vitais $(\mathrm{r}=0,26 ; \mathrm{p}=0,02)$. Além disso, a presença de sintomas de estresse foi associada ao maior número de eventos vitais $(\mathrm{r}=0,37 ; \mathrm{p}=0,001)$ e a menor idade materna $(\mathrm{r}=0,26 ; \mathrm{p}=0,02)$.

Essas variáveis foram, portanto, incluídas no modelo de predição da enxaqueca em mulheres, analisado por meio da regressão logística, sendo consideradas como variáveis preditoras para a variável predita (presença ou não de enxaqueca). A presença de estresse foi a única variável preditora que permaneceu no modelo com poder de explicação da presença de enxaqueca em mulheres $(\beta=1,67$; $\mathrm{ER}=0,55 ; \mathrm{p}=0,002 ; \mathrm{OR}=5,34 ; \mathrm{IC}=1,80-15,82)$. A presença de estresse aumentou em 5,34 vezes mais a chance de as mulheres apresentarem enxaqueca.

\section{Discussão}

O presente estudo teve por objetivo investigar a relação entre enxaqueca (migrânea) em mulheres e estresse, levandose em conta variáveis pessoais e do contexto ambiental. Para responder a esse objetivo serão discutidos primeiramente, os achados acerca da presença de enxaqueca e de estresse em mulheres, bem como da associação dessas duas variáveis e, por fim, o modelo de predição da presença de enxaqueca.

Em relação à prevalência de enxaqueca nas mulheres, o presente estudo encontrou que mais da metade $(55 \%)$ apresentaram sintomas de enxaqueca, segundo o teste da Sociedade Brasileira de Cefaleia. Destaca-se que estudos anteriores verificaram alta prevalência de enxaqueca em mulheres (Bugdayci et al, 2005; Mascella, 2011; Rasmussen, 2001; Stewart, Simon, Schecheter, \& Lipton, 1995) devido a diversos fatores, especialmente aos relacionados a hormônios femininos. Entretanto, cabe ressaltar que, variações acerca das prevalências de enxaqueca podem estar relacionadas à diversidade de métodos de avaliação utilizados, à variação da idade da amostra dos estudos e às características dos respondentes do instrumento de avaliação da dor (Annequin, Tourniaire, \& Massiou, 2000), além do fato de que as mulheres procuram mais ajuda médica quando comparadas aos homens (Krymchantowski, 2008).

No presente estudo, a prevalência do estresse em mulheres (59\%) foi maior do que a prevalência de enxaqueca. Este achado está parcialmente de acordo com estudos anteriores, uma vez que a prevalência da presença de estresse encontrada em mulheres no presente estudo ficou um pouco abaixo quando comparado com outros estudos, os quais obtiveram prevalências de estresse entre 84 a 90\% (Galego, 2006; Lipp, Malagris, \& Novais, 2007; Mascella, 2011; Tanganelli, 2000). De qualquer modo, verifica-se que os níveis de estresse em mulheres têm se mantido elevados.

Ainda em relação ao estresse, a maior parte das mulheres encontrava-se na fase de resistência, que corresponde a uma fase na qual o organismo está agindo para impedir o desgaste total de energia e tentando restabelecer a homeostase. Verifica-se que nessa fase há uma maior produção de cortisol, que em altos níveis deixa o organismo mais vulnerável a vírus e bactérias (Lipp, 2000). Quanto à predominância dos sintomas de estresse, verifica-se a prevalência dos sintomas psicológicos sobre os físicos. Ambos os achados são sustentados pelos estudos de Tanganelli (2000), Galego (2006), Lipp et al. (2007) e Mascella (2011).

$\mathrm{O}$ maior número de eventos vitais foi associado à presença de estresse. Segundo Lipp (2000), o estressor é considerado qualquer evento que amedronte, confunda ou excite a pessoa e ainda, ocasione uma quebra da homeostase interna do organismo, exigindo uma resposta do organismo, em forma de adaptação ou ajustamento ao ambiente. Destacase ainda que, um maior o número de eventos estressantes potencializam a condição de risco biopsicossocial a que o indivíduo é exposto, tornando-o mais vulnerável a outros estressores. Isso sugere que os eventos de vida em si podem levar ao estresse patológico. No entanto, cabe ressaltar que o estressor poderá ser considerado benéfico ou maléfico mediante a avaliação que o indivíduo faz das situações pelas quais passa (Lazarus \& Folkman, 1984). Além disso, diferenças orgânicas, psicológicas e ambientais têm um importante papel moderador na avaliação dos eventos causadores de estresse e tendem a variar de indivíduo para indivíduo.

A associação entre sintomas de enxaqueca e estresse apresentada no presente estudo também é apontada na literatura. Mascella (2011) verificou que mulheres com enxaqueca, além de apresentarem estresse nas fases onde há um maior agravamento dos sintomas (fase de quaseexaustão), também apresentaram altos níveis de ansiedade e depressão quando comparadas a mulheres com outros tipos de dor de cabeça.

No presente estudo, a chance de as mulheres terem enxaqueca mostrou-se aumentada em cinco vezes na presença de estresse. Segundo Lipp (2005), muitas vezes o estresse e a dor encontram-se intimamente associados, pois o estresse pode aparecer tanto como desencadeador ou concorrente para a etiologia da dor ou pode ocorrer em consequência da dor. Além disso, a relação entre estresse e dor baseia-se na hipótese destes fatores induzirem reações fisiológicas e psicológicas. O estresse pode ainda aumentar, perpetuar ou reduzir a capacidade de enfrentamento à dor do indivíduo (Barr, 1994).

Em conclusão, as mulheres do presente estudo apresentaram-se vulneráveis e constituem-se em um grupo em risco para um desequilíbrio emocional, devido a dois fatores: a) pela presença de enxaqueca, que corresponde à mais incapacitante das dores de cabeça; b) pela presença de estresse, na fase de resistência, que corresponde a uma fase em que se inicia o agravamento de sintomas negativos de estresse e com predominância de sintomas psicológicos. 
Dessa forma, mulheres com enxaqueca e estresse associados necessitam de uma melhor atenção em relação ao tratamento para enxaqueca e/ou estresse, que proporcione mais qualidade de vida e bem-estar a elas.

Destaca-se que o ponto forte do estudo é a exclusão dos antecedentes psiquiátricos e a inclusão da análise dos eventos vitais como variáveis de controle na relação entre enxaqueca e estresse em mulheres. A primeira poderia ser uma variável de confusão interferindo nos achados obtidos, enquanto a segunda apresenta forte associação com estresse e que, portanto, precisava ser incluída na análise.

No entanto, algumas limitações do estudo devem ser apontadas. Em relação à identificação de indicadores de enxaqueca em mulheres, não houve um diagnóstico neurológico, entretanto, o teste utilizado foi elaborado pela Sociedade Brasileira de Cefaleia (Speciali, 2003) e permite a verificação da intensidade, duração, frequência e características de sintomas relacionados à enxaqueca. Entretanto, como salientado pela literatura, esses sintomas estão fortemente associados (Barr, 1994), sendo necessário um delineamento mais específico para verificar o efeito do estresse na enxaqueca em mulheres. Estudos futuros podem considerar esse tipo de delineamento que compare mulheres com e sem estresse e a presença de enxaqueca.

\section{Referências}

Annequin, D., Tourniaire, B., \& Massiou, H. (2000). Migraine and headache in childhood and adolescence. Pediatric Clinics of North America, 47(3), 617-631.

Associação Brasileira de Empresas de Pesquisa (ABEP) (2013). Critério padrão da classificação econômica Brasil/2013. Retrieved from http://www.abep.org/novo/ Content.aspx?ContentID $=835$.

Aromaa, M., Rautava, P., Helenius, H., \& Sillanpää, M. L. (1998). Factors of early life as predictors of headache in children at school entry. Headache, 38, 23-30.

Barr, R. (1994). Pain experiences in children: Developmental and clinical characteristics. In P. D. Wall, \& R. Melzack (Eds.), Textbook of pain (3a ed., pp. 739-765). Edinburgh: Churchill.

Bosco, A. (2006). Cefaléia em crianças- Sinal de vulnerabilidade ao stress: Estudo comparativo em dois hospitais públicos. (Unpublished doctoral dissertation). Universidade Estadual de Campinas, Campinas.

Bugdayci, R., Ozge, A., Sasmaz, T., Kurt, A.O., Kaleagasi, H., Karakelle, A., . . . Siva, A. (2005). Prevalence and factors affecting headache in Turkish schoolchildren. Pediatrics International, 47, 316- 322.

Campo, J. V., Comer, D. M., Jansen-Mcwilliams, L., Gardner, W., \& Kelleher, K. J. (2002). Recurrent pain, emotional distress, and health service use in childhood. The Journal of Pediatrics, 141, 76-83.

Cathcart, S., Winefield, A.H., Lushington, K., \& Rolan, P. (2010). Stress and tension-type headache mechanisms. Cephalalgia, 30(10), 1250-1267.
Del Ben, C. M. (1995). Estudo da confiabilidade do diagnóstico psiquiátrico obtido através da Entrevista Clínica Estruturada para o DSM-III-R (SCID) em serviço ambulatorial de um hospital escola. (Unpublished master's thesis). Universidade de São Paulo, Ribeirão Preto.

Domingues, R. B., Aquino, C. C. H, Santos, J. G., Silva, A. L. P., \& Kuster, G. W. (2006). Prevalence and impact of headache and migraine among pomeranians in Espírito Santo, Brazil. Arquivos de Neuropsiquiatria, 64(4), 954- 957.

Fearon, P., \& Hotopf, M. (2001). Relation between headache in childhood and physical and psychiatric symptoms in adulthood: National birth cohort study. British Medical Journal, 322(12), 1-6.

Fernandes, L. C. (2004). Estudo epidemiológico populacional da prevalência de cefaléia na cidade de Ribeirão Preto. (Unpublished master's thesis). Universidade de São Paulo, Ribeirão Preto.

Galego, J. C. B. (2006). Cefaléia crônica diária: Classificação, estresse e impacto sobre a qualidade de vida. (Unpublished doctoral dissertation). Faculdade de Medicina de São José do Rio Preto, São José do Rio Preto.

Göbel, H. (2001). Classification of headaches. Cephalalgia, 21, 770- 773.

Guidetti, V., \& Galli, F. (1998). Evolution of headache in childhood and adolescence: An 8- year follow-up. Cephalalgia, 18(7), 449-454.

Headache classification subcommittee of the International Headache Society. The International classification of headache disorders. (2004). Cephalalgia, 24(suppl 1), 9-160.

Holmes, T. H., \& Rahe, R. (1967). The Social Readjustment Rating Scale. Journal of Psychosomatic Research, 11, 213-218.

International Headache Society (2008). The classification. Retrieved from http://www.i-h-s.org/

Kanner, A. D., Coyne, J. C., Schaefer, C., \& Lazarus, R. S. (1981). Comparison of two models of stress measurement: Daily hassles and uplifts versus major life events. Journal of Behavioral Medicine, 4, 1-39.

Karwautz, A., Wober-Bingol, C., \& Wober, C. (1993). Idiopathic headache in childhood and adolescence. Journal: Nervenarzt, 64 (12), 753-765.

Kienbacher, C., Wöber, C., Zesch, H. E., Hafferl-Gattermayer, A., Posch, M., Karwautz, A., . . Wöber-Bingöl, Ç. (2006). Clinical features, classification and prognosis of migraine and tension-type headache in children and adolescents: a long-term follow-up study. Cephalalgia, 26(7), 820-830.

Krymchantowski, A. V. (2008). Condutas em cefaléia: Avaliação e tratamento. São Paulo: Lippincott Williams \& Wilkins.

Lazarus, R., \& Folkman, S. (1984). Stress, appraisal and coping. New York: Springer.

Lipp, M. E. N. (2000). Inventário de sintomas de stress para adultos de Lipp (ISSL). São Paulo: Casa do Psicólogo.

Lipp, M. E. N. (Ed.) (2004). Stress no Brasil: Pesquisas avançadas. Campinas: Papirus.

Lipp, M. E. N. (2005). Stress e o turbilhão da raiva. São Paulo: Casa do Psicólogo.

Lipp, M. E. N., Malagris, L. E. N., \& Novais, L. N. (2007). Stress ao longo da vida. São Paulo: Casa do Psicólogo. 
Lipp, M. E. N., \& Malagris, L. E. N. (2001). Stress: Aspectos históricos, teóricos e clínicos. In B. Rangé (Ed.), Psicoterapia cognitivo-comportamentais: Um diálogo com a psiquiatria. (pp. 475-490). Porto Alegre: Ed. Artmed.

Mascella, V. (2011). Stress, sintomas de ansiedade e depressão na migrânea e cefaléia tensional. (Unpublished master's thesis). Pontifícia Universidade Católica de Campinas, Campinas.

Nobre, M. E. (2006). Cefaléia em salvas. São Paulo: Lemos Editorial.

Organização Mundial de Saúde (2006). Benefits of physical activity. Retrieved from http://www.who.int/mediacentre/factsheets/ fs277/en/.

Puccini, R. F., \& Bresolin, A. M. B. (2003). Dores recorrentes na infância e adolescência. Jornal de Pediatria, 79(1), 65-76.

Queiroz, L. P., Barea, L. M., \& Blank, N. (2006). An epidemiological study of headache in Florianópolis, Brazil. Cephalalgia, 26, 122-127.

Queiroz, L. P., Peres, M. F., Piovesan, E. J., Kowacs, F., Ciciarelli, M. C., Souza, J. A., \& Zukerman, E. (2009). A nationwide population-based study of tension-type headache in Brazil. Headache, 49(1), 71-78.

Rasmussen, B. K. (2001). Epidemiologia da cefaléia. Cephalalgia, 21, 774- 777.

Savoia, M. G. (1999). Escalas de eventos vitais e de estratégias de enfrentamento. Revista de Psiquiatria Clínica, 26(2), 57-67.
Selye, H. A. (1952). The story of the adaptation syndrome. Montreal: Act Inc Med. Publ.

Soares, N. E., \& Fernandes, L. M. (1989). A medida do nível sócio- econômico cultural. Arquivos Brasileiro de Psicologia, 41(2), 35-43.

Speciali, J. G. (2003). Entendendo a enxaqueca. Ribeirão Preto: FUNPEC Editora.

Spitzer, R. L., Williams, J. R., Gibbon, M., \& First, M. B. (1989). Instruction manual for the structured clinical interview for DSM III - R (SCID, 5/11/89 Revision). Biometrics Research Department. New York State Psychiatric Institute.

Stewart, W. F., Lipton, R. B., Celentano, D. D., \& Reed, M. L. (1992). Prevalence of migraine headache in the United States: Relation to age, income, race, and other socio demographic factors. The Journal of the American Medical Association, 267(1), 64-69.

Stewart, W. F., Simon, D., Schechter, A., \& Lipton, R. B. (1995). Population variation in migraine prevalence: A meta-analysis. Journal of Clinical Epidemiology, 48(2), 269-280.

Stovner, L. J., Hagen, K., Jensen, R., Katsarava, Z., Lipton, R., Scher, A., . . Z Zwart, J. A. (2007). The global burden of headache: A documentation of headache prevalence and disability worldwide. Cephalalgia, 27, 193-210.

Tanganelli, M. L. S. (2000). Mulher chefe de família: Perfil, estudo e tratamento do stress. (Unpublished doctoral dissertation). Pontifícia Universidade Católica de Campinas, Campinas. 